Journal Club

Editor's Note: These short, critical reviews of recent papers in the Journal, written exclusively by graduate students or postdoctoral fellows, are intended to summarize the important findings of the paper and provide additional insight and commentary. For more information on the format and purpose of the Journal Club, please see http://www.jneurosci.org/misc/ifa_features.shtml.

\title{
Performance Monitoring in Realistic Environments: Can Translating Neuroscientific Insights Augment Real-World Behavioral Adaptation?
}

\author{
Jan R. Wessel \\ Psychology Department, University of California, San Diego, La Jolla, California 92093 \\ Review of Torrecillos et al.
}

To exert successful goal-directed behaviors, humans must continually monitor their actions for deviations from their intended outcomes. If a mismatch between the intended and actual outcome of an action is detected, performance monitoring enables the concurrent adaptation of ongoing behavior. One commonplace realworld example of this is driving a car, where both action slips (e.g., shifting into the wrong gear), as well as sudden, unanticipated events (e.g., a failing power steering) can necessitate quick behavioral adjustments, which ensure that the intended goal (driving safely) is reached. Normal aging, as well as some neuropsychiatric disorders (e.g., obsessive-compulsive disorder and attention-deficit hyperactivity disorder) can lead to deteriorations in performance monitoring, which can severely impair goal-directed behaviors in everyday life.

The cognitive neuroscience of performance monitoring offers unique insights into how monitoring and adaptation of behavior is implemented in the brain. One ultimate goal of this branch of neuroscience is to develop methods that

Received May 2, 2014; revised May 27, 2014; accepted June 3, 2014.

I thank Kristi Hendrickson and J. Bruce Morton for helpful comments on this Journal Club.

Correspondence should be addressed to Dr. Jan R. Wessel, University of California, San Diego, Psychology Department, 3133 McGill Hall, 9500 Gilman Drive, La Jolla, CA 92103. E-mail: jwessel@ucsd.edu.

DOI:10.1523/JNEUROSCI.1840-14.2014

Copyright $\odot 2014$ the authors $\quad 0270-6474 / 14 / 348934-03 \$ 15.00 / 0$ could assist successful goal-directed behavior in real-world situations, particularly in populations with impaired performance monitoring. Critically, the first step in such a translation from basic science to the real world is to test hypotheses in increasingly realistic experiments that accurately mimic the complex cognitive demands of everyday life.

Torrecillos et al.'s (2014) recent paper, published in The Journal of Neuroscience, is an outstanding example of such a first step. The authors developed a task in which human participants performed reaching movements while their arms were fixed in a force-feedback exoskeleton, which controlled the mechanical resistance against the planned reaching movements. By unpredictably varying the degree of mechanical resistance on a subset of trials, the authors introduced action errors of varying magnitude into participants' movements. Experiencing such unexpected resistance during an arm movement is very similar to the example of a car's suddenly failing power steering, which was used above to illustrate the utility of performance monitoring in a realistic situation.

Using scalp electroencephalography (EEG), Torrecillos et al. (2014) identified a component in the event-related potential (ERP) that was increased following such unexpected mechanical perturbations. This component, which the authors termed ERP-K (K for "kinematic error"), was characterized by a negative voltage deflection with frontocentral distribution. The amplitude of the ERP-K parametrically varied according to the magnitude of the mechanical resistance (and/or the magnitude of the resulting deviations from the intended hand path).

To elucidate the exact mechanism underlying the ERP-K, the authors used temporal independent component analysis (ICA). ICA is used to identify independent neural source components that underlie the composite EEG signal mixture measured on the scalp. Scalp EEG measures a mixture of activity emitting from separate neuronal dipole fields, which correspond to individual brain areas/cortical domains involved in ongoing neural computations (Onton et al., 2006). To recover the activity of these effective EEG sources, ICA transforms the observed time courses (i.e., activity measured at scalp electrodes) into maximally independent components, under the assumption that computations in separate cortical domains have (mathematically) independent time course profiles over time. By maximizing the independence of time courses (through iterative data transformations that minimize mutual information), ICA aims to uncover the true, independent cortical sources of the observed EEG mixture on the scalp. Once these sources are identified, it can be tested whether neural computations on 
two different types of trials result from the activity of the same EEG source/cortical domain. Using this technique, the authors showed that the ERP-K was generated by the same cortical domain (i.e., the same independent signal component) that underlies the feedback-related negativity (FRN; Miltner et al., 1997). The FRN is a frontocentral negativity as well, and it is observed following negative performance feedback. In the current study, it was evoked by negative visual feedback ("Too slow!") regarding the speed of the reaching movement.

Critically, the cortical domain that generates the FRN and ERP-K was found to generate other frontocentral ERP negativities in previous studies that used this ICA-based technique. These negativites are related to different aspects of performance monitoring. For example, the same cortical domain that generates the FRN has been shown to underlie the error-related negativity (ERN; Gentsch et al., 2009), which is observed after simple action errors. Furthermore, the noveltyN2, a frontocentral negativity evoked by unexpected (but valence-neutral) action outcomes, is also generated by the same cortical domain (Wessel et al., 2012). Importantly, FRN, ERN, and novelty-N2 have not only been shown to be related to the monitoring for negative feedback, errors, or unexpected outcomes, respectively, but also to the amount of adaptive behaviors that follow such events. The FRN's amplitude has been shown to predict behavioral change following negative feedback (Cohen and Ranganath, 2007), the amplitude of the ERN has been shown to predict reaction time slowing following action errors (Debener et al., 2005), and the novelty-N2 has been proposed to signal the need for processing of unexpected perceptual events (Daffner et al., 2000).

Already, these studies hint at the wide applicability of frontocentral ERP negativities in many different scenarios in which ongoing behavior needs to be monitored and adapted. However, Torrecillos et al.'s (2014) study enriches this picture in a crucial way. The vast majority of studies of performance monitoring (including those referenced in the previous paragraph) have used simple experimental designs, such as binary forced-choice reaction times paradigms. While such simple paradigms offer tight stimulus control, and hence are of high internal validity, they have little in common with most realistic scenarios in which action control is needed outside of the laboratory. Real-world scenarios rarely have bi- nary outcomes, and only few actions are as simple as pushing a single button on a keyboard. The force-impacted reaching movements performed in Torrecillos et al.'s (2014) study, however, are of remarkably high ecological validity, as discussed above. The authors' elaborate task design enabled them to simulate a realistic scenario in which performance monitoring and behavioral adaptation are necessary, while simultaneously maintaining a high degree of stimulus control.

The use of ICA to analyze their neural data provides two additional advantages. First, ICA provides direct evidence of the fact that two seemingly unrelated neural processes like the ERP-K and the FRN are generated by the same cortical domain. This makes it possible to generate hypotheses about one process from preexisting knowledge about the other. In other words, since past research showed that performance-monitoring-related ERP components like the FRN, ERN, and novelty-N2 are often directly predictive of adaptive behaviors, the same prediction can be derived for the ERP-K, which shares a common neural domain of generators with those ERP components. Second, ICA enables the investigation of the source signal of interest separately from activity of extraneous source signals, thereby increasing the signal-to-noise ratio (SNR) of the relevant signal. Increased SNR facilitates relating brain signals (e.g., the ERP-K) to other variables (e.g., adaptive behaviors) on a trial-by-trial level. Hence, the usage of ICA provides both the means and motivation for future research on the role of negative frontocentral ERP components (like the ERP-K) in realistic situations that demand performance monitoring and behavioral adaptation.

Together, the authors' combination of mechanical engineering in task design and state-of-the-art techniques in neural data analysis allowed the translation of basic research findings about performance monitoring into a highly realistic experimental situation. Such interaction between engineering and neuroscience is pivotal to developing methods that could increase the efficacy of cognitive control in real-world situations. Some potential approaches are already within reach. Recently, brain stimulation techniques have shown promise in increasing performance monitoring and/or behavioral adaptation. In one example, frontal transcranial direct-current stimulation (tDCS) was used to influence the amplitude of the ERN, which resulted in corresponding changes of adaptive behaviors following action errors (Reinhart and Woodman, 2014). Furthermore, frontal tDCS can restore conscious error perception in older adults, which has been proposed to be a precursor of the implementation of adaptive behaviors (Harty et al., 2014). The success of such approaches, combined with the translational efforts described in Torrecillos et al.'s (2014) study, raise the hope that eventually, impairments in real-world performance monitoring and behavioral adaptation could be alleviated using augmentative brain stimulation.

Furthermore, identifying neural signatures of performance monitoring in realistic scenarios is a potential first step in using such signals in brain-computer interfaces (BCI). While this scenario may seem like science fiction, modern BCI technology has helped humans to restore motor and sensory function, and could help to alleviate deficits in cognitive control as well. BCI technology could help humans to assess whether the amount of performance-monitoring brain activity is sufficient given acute situational demands on cognitive control. Because of their wide applicability, low latency, and high signal-to-noise ratio (especially when buttressed by ICA-based techniques), the frontocentral negativities Torrecillos et al. (2014) and others have described constitute an ideal input signal for such a brain-computer interface. Clearly, the objective demand of behavioral adaptation is not always as obvious as in the example of a malfunctioning power steering. Yet, in examples like that, in which optimal and suboptimal outcomes are very narrowly circumscribed, a BCI approach could offer small but effective safeguards to ensure that behavioral adaptations are implemented when of large importance.

In summary, the study by Torrecillos et al. (2014) demonstrates that even in realistic scenarios, EEG can provide a low-latency measurement of the performance-monitoring process. In the future, cognitive neuroscientists should attempt to leverage that knowledge to develop effective tools that augment performance monitoring in everyday situations. The combination of such tools with modern brain-stimulation methods could ultimately result in therapeutic devices targeted at supporting successful goaldirected behavior in populations affected by pathological or declining performance monitoring. 


\section{References}

Cohen MX, Ranganath C (2007) Reinforcement learning signals predict future decisions. J Neurosci 27:371-378. CrossRef Medline

Daffner KR, Scinto LF, Calvo V, Faust R, Mesulam MM, West WC, Holcomb PJ (2000) The influence of stimulus deviance on electrophysiologic and behavioral responses to novel events. J Cogn Neurosci 12:393-406. CrossRef Medline

Debener S, Ullsperger M, Siegel M, Fiehler K, von Cramon DY, Engel AK (2005) Trial-by-trial coupling of concurrent electroencephalogram and functional magnetic resonance imaging identifies the dynamics of performance monitoring. J Neurosci 25:11730-11737. CrossRef Medline

Gentsch A, Ullsperger P, Ullsperger M (2009)
Dissociable medial frontal negativities from a common monitoring system for self- and externally caused failure of goal achievement. Neuroimage 47:2023-2030. CrossRef Medline

Harty S, Robertson IH, Miniussi C, Sheehy OC, Devine CA, McCreery S, O'Connell RG (2014) Transcranial direct current stimulation over right dorsolateral prefrontal cortex enhances error awareness in older age. J Neurosci 34:3646-3652. CrossRef Medline

Miltner WH, Braun CH, Coles MG (1997) Event-related brain potentials following incorrect feedback in a time-estimation task: evidence for a "generic" neural system for error detection. J Cogn Neurosci 9:788-798. CrossRef Medline

Onton J, Westerfield M, Townsend J, Makeig S (2006) Imaging human EEG dynamics using independent component analysis. Neurosci Biobehav Rev 30:808-822. CrossRef Medline

Reinhart RM, Woodman GF (2014) Causal control of medial-frontal cortex governs electrophysiological and behavioral indices of performance monitoring and learning. J Neurosci 34:4214-4227. CrossRef Medline

Torrecillos F, Albouy P, Brochier T, Malfait N (2014) Does the processing of sensory and reward-prediction errors involve common neural resources? Evidence from a frontocentral negative potential modulated by movement execution errors. J Neurosci 34:4845-4856. CrossRef Medline

Wessel JR, Danielmeier C, Morton JB, Ullsperger M (2012) Surprise and error: common neuronal architecture for the processing of errors and novelty. J Neurosci 32:7528-7537. CrossRef Medline 